\title{
Advances in Shallow Landslide Hydrology and Triggering Mechanisms: A Multidisciplinary Approach
}

\author{
Claudia Meisina ${ }^{(D},{ }^{1}$ Marco Bittelli, ${ }^{2}$ Roberto Valentino, ${ }^{3}$ Massimiliano Bordoni, ${ }^{1}$ \\ and Roberto Tomás-Jover ${ }^{4}$ \\ ${ }^{1}$ Department of Earth and Environmental Sciences, University of Pavia, Via Ferrata 1, 27100 Pavia, Italy \\ ${ }^{2}$ Department of Agricultural Sciences, University of Bologna, Viale Fanin 44, 40127 Bologna, Italy \\ ${ }^{3}$ Department of Chemistry, Life Sciences and Environmental Sustainability, University of Parma, Parco Area delle Scienze, 157/a, \\ 43124 Parma, Italy \\ ${ }^{4}$ Departamento de Ingeniería Civil, Universidad de Alicante, Apartado de Correos 99, 03080 Alicante, Spain
}

Correspondence should be addressed to Claudia Meisina; claudia.meisina@unipv.it

Received 16 June 2019; Accepted 16 June 2019; Published 27 November 2019

Copyright ( 92019 Claudia Meisina et al. This is an open access article distributed under the Creative Commons Attribution License, which permits unrestricted use, distribution, and reproduction in any medium, provided the original work is properly cited.

The vadose zone of steep slopes is often affected by rainfallinduced shallow landslides, which can cause widespread direct and indirect damage to the terrain and infrastructures, as well as urban and rural developments. These phenomena are determined by hydrological or subsurface flow processes and also mechanical (stress equilibrium) processes. Some models attempt to link dynamics of hydrologic behavior with the mechanical state of a hillslope and the onset of failure. However, the hydrological dynamics leading to shallow landslide initiation, the hydraulic properties at the slope scale, and the role of hysteretic effects as well as the soil nonequilibrium processes in slope stability assessment are still not completely understood and require further investigation. Furthermore, these open questions are generally treated separately by geologists, hydrologists, agronomists, and geotechnical engineers, whereas a multidisciplinary approach is a key factor in the study of these phenomena occurring in the vadose zone.

Starting from these issues, the main focus of this special issue is on presenting the advances in shallow landslide hydrology from both the earth sciences and soil mechanics perspectives and their influence on behavior and triggering of shallow landslides.

This special issue collects papers representing some of the most recent developments in this field, with a special emphasis given to recent results obtained by both smallscale experiments and analysis of specific case histories. The main topics of the papers included in the special issue regarded the following:

(i) Analysis and modeling of landslide hydrology at different scales

(ii) Hydrological modeling of the materials forming deposits of past landslides

(iii) Field hydrological monitoring of slopes

(iv) Seasonal, yearly, and interyearly hydrological dynamics of a slope

(v) Models of hydromechanical triggering of shallow landslides

(vi) Antecedent and initial hydrological conditions that predispose to landslide triggering

(vii) Porous media dynamics in landslide systems

(viii) Landslide water balance models

Ten papers were submitted for this special issue. Our distinguished reviewers from respective research fields 
narrowed the field to six papers which were finally accepted. The following is a short summary of the findings of each of these papers.

C. Zhang et al. performed physical tank experiments to investigate the ways of generation of pore water pressure in a slope affected by a landslide. The results of this experimental approach stress that pore water pressure values and changes in time, related to the different positions of the groundwater table along the hillslope, are significantly affected by infiltration time from the surface to different layers in depth, by surface runoff amount, and by lateral flows.

L. Schilirò et al. investigated the impact of the initial soil hydrological conditions on the triggering of rainfallinduced shallow landslides, proposing an innovative experimental setup which integrated laboratory simulations by means of a sloping flume and physically based numerical modeling. Initial hydrological conditions have a fundamental role on determining the type of the triggering mechanism and the soil types that could be affected by shallow failures.

H. Cai et al. analyzed the hydromechanical behavior of the materials forming the accumulation zone of a past earthquake-induced landslide, which was affected by erosions and shallow failures. Monitoring of water content and pore water pressure trends and of the flow directions in a laboratory small-scale physical model of the real landslide allowed recognizing preferential flows and seepage-induced internal erosion in the deposit that could lead to the triggering of shallow instabilities.

A. Wayllace et al. analyzed the hydrological behavior of an active landslide in Colorado (USA) and its effects on the stability of the slope. Field observation of displacements and groundwater variations are used to determine a conceptual model that takes into account also site morphology and stratigraphy, atmospheric conditions, and the main physical mechanisms in the hillslope. A series of 2D finite element numerical simulations, which are based on the conceptual model and calibrated with field data, are then used to assess slope stability.

J. Iqbal et al. analyzed the effect of reservoir fluctuations on an active landslide in the Xiangjiaba Reservoir area, Southwest China. On the basis of field investigations and laboratory tests, the authors carried out a slope stability analysis using FLAC 3D software. Numerical results shed light on the fact that the minimum safety requirements under the working condition of reservoir level fluctuation are not reached and then surface monitoring should be carried out in order to set up early warning prior to failure.

Y.-S. Yang and H.-F. Yeh evaluated the probability of failure by means of a fuzzy point estimate method in order to consider the inherent uncertainties associated with soil properties (i.e., cohesion and friction angle). To this aim, a hydromechanical coupling model on HYDRUS 2D and the Slope Cube Module were used. The results reveal that fuzzy theory effectively evaluates the variability of the factor of safety as well as the reliability index.

\section{Conflicts of Interest}

I confirm that the Lead Guest Editor and the other Guest Editors have no conflict of interest.

Claudia Meisina

Marco Bittelli

Roberto Valentino

Massimiliano Bordoni

Roberto Tomás-Jover 

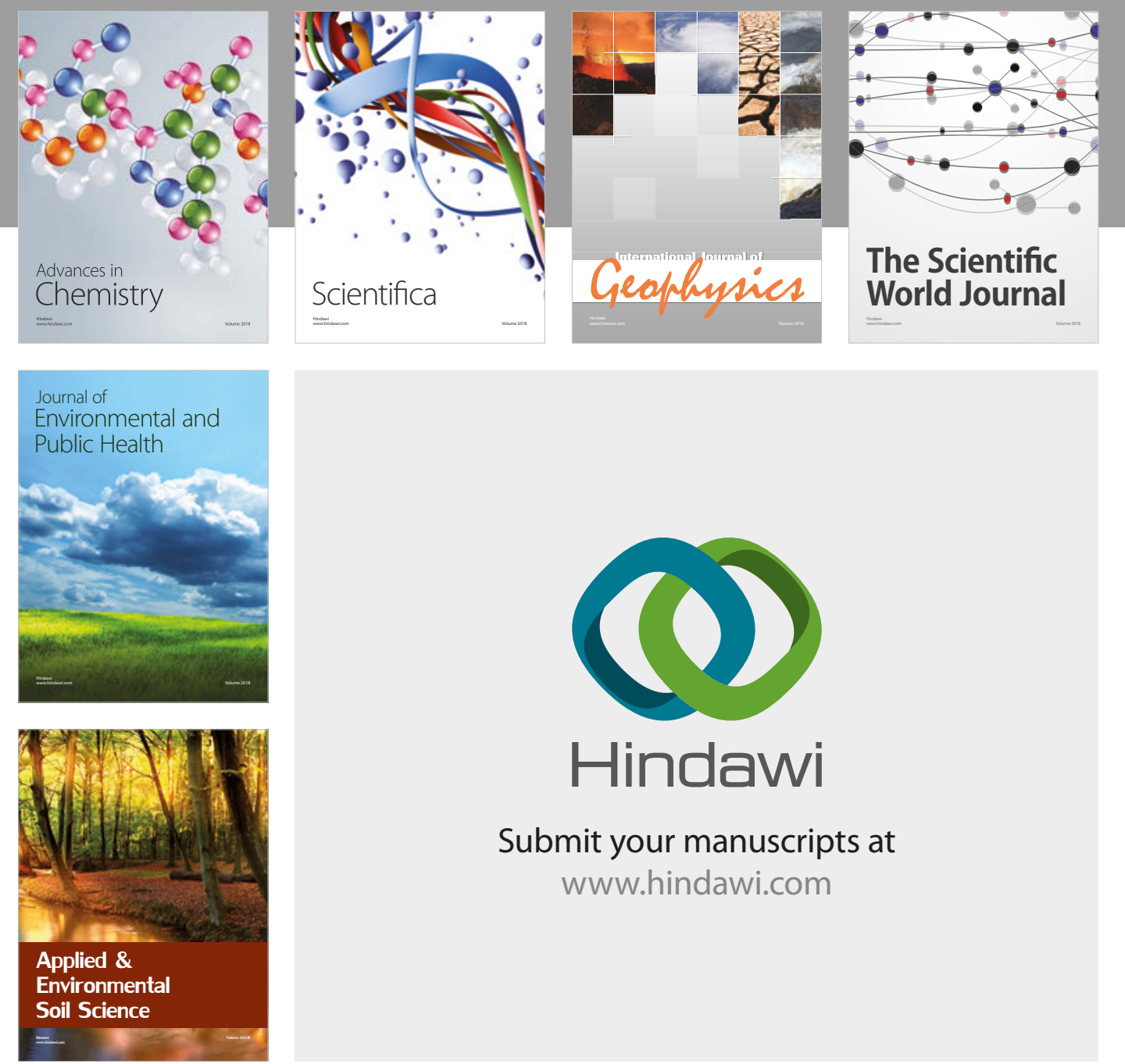

The Scientific

\section{World Journal}
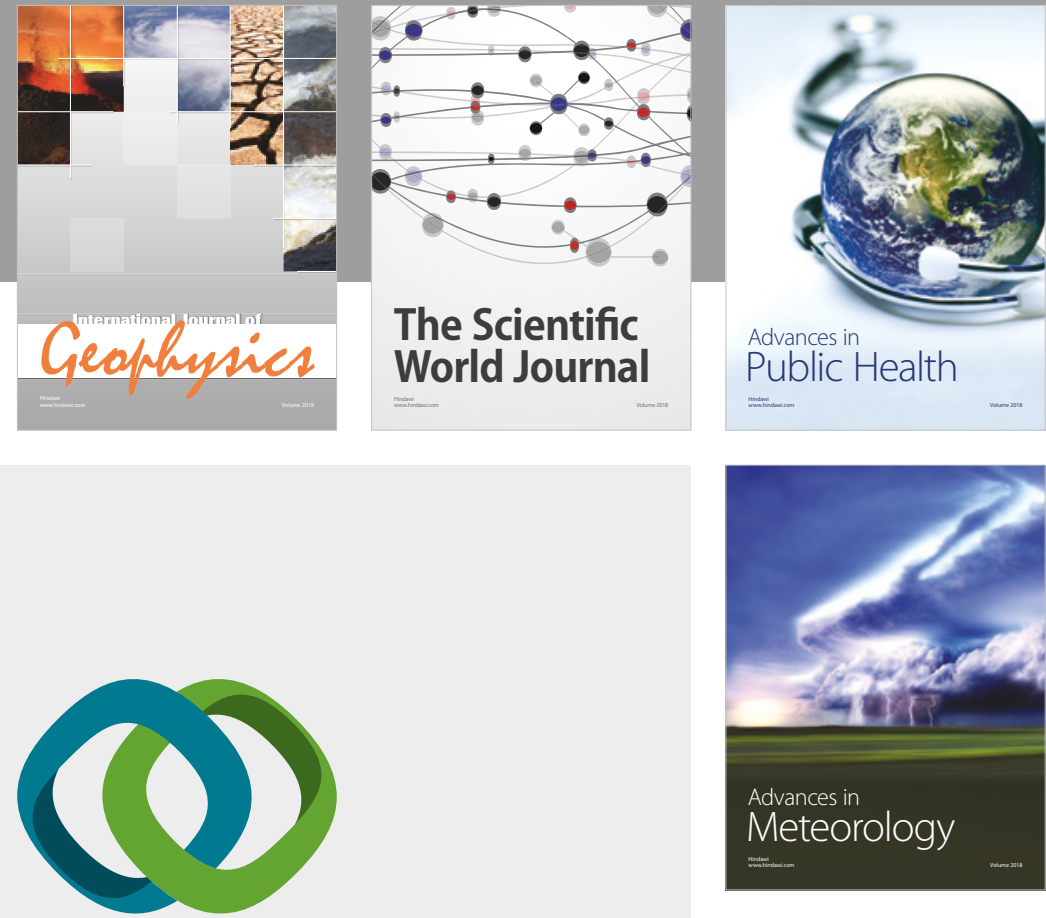

Advan

Public Health

\section{Hindawi}

Submit your manuscripts at

www.hindawi.com
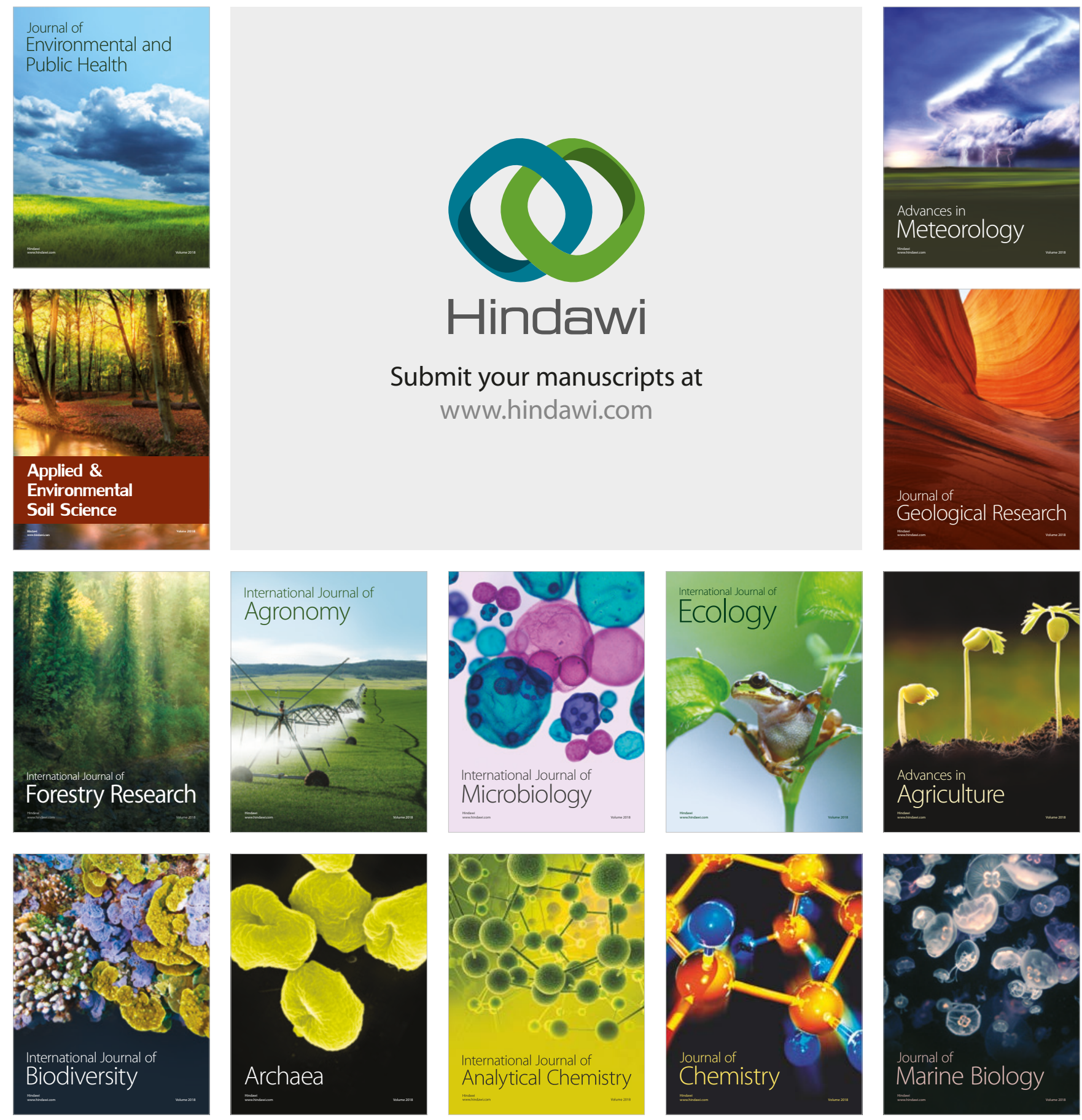